\title{
Effect of Cigarette Smoking on Human Gingival Mucosa-Histological and Morphometric Study
}

\section{Ghada Galal Hamam *and Dalia Alaa El-Din Aly El-Waseef}

Department of Histology and Cell Biology, Faculty of Medicine, Ain Shams University, Cairo, Egypt

*Corresponding author: Hamam GG, Department of Histology and Cell Biology, Faculty of Medicine, Ain Shams University, Cairo, Egypt, Tel: 0020 01003960601; Email: ghada.hamam@yahoo.com

Received date: July 30, 2018; Accepted date: August 14, 2018; Published date: August 20, 2018

Copyright: ( 2018 Hamam GG, et al. This is an open-access article distributed under the terms of the Creative Commons Attribution License, which permits unrestricted use, distribution, and reproduction in any medium, provided the original author and source are credited.

\begin{abstract}
Introduction: Cigarette Smoking is considered the most preventable cause of diseases and premature deaths in the history of mankind. It is associated with high levels of morbidity and mortality all over the world.
\end{abstract}

Aim of the study: To study the light and electron microscopic structure of clinically normal gingival mucosa in nonsmoker versus smoker adult males.

Subjects and methods: Eighteen adult males (volunteers) were divided according to the intensity and duration of smoking (pack year index) into three groups. Group I (non-smokers control), group II (pack year index 1-9) and group III (pack year index >10). Specimens from the gingival mucosa were obtained and processed for proper histological techniques. Paraffin sections were stained with H\&E, Mallory`s trichrome stain and immunohistochemical reaction for caspase-3. Transmission electron microscopic study, morphometric and statistical analysis were also performed.

Results: Basal cell hyperplasia, increase thickness of epithelium, apoptosis of epithelial cells, and inflammatory cellular infiltration in the lamina propria, desmosomal disruption, increase glycogen granules and melanin pigments were noticed in group II and were more increased in group III.

Conclusions: Clinically normal gingival mucosa of cigarette smokers shows light and electron microscopic structural changes that are directly related to the pack year index of the individual.

Keywords: Cigarette smoking; Gingival mucosa; Caspase-3; Electron microscopy; Histology; Immunohistochemistry

\section{Introduction}

Tobacco smoking is a global problem of civilization; with the estimated number of tobacco smokers about 1.3 billion. Statistically, the life expectancy of smokers is 15 years less than that of non-smokers [1]. Cigarette smoke increases the susceptibility to oral mucosal infection. It is a well-recognized risk factor for periodontitis, oral candidiasis and oral leukoplakia $[2,3]$.

The use of tobacco and its related products are still very prevalent, although it is an established fact that they are one of the leading causative agents for oral cancer. Oral cancer is one of the most debilitating diseases affecting men [3]. It was reported that almost 263,020 oral cavity cancers and 127,654 oral cancer deaths occur worldwide each year. Individuals at high risk of developing oral cancer are mainly older, males, heavy tobacco smokers and alcohol users, and those with poor diet and low socioeconomic status [4].

Tobacco smoke contains more than 4000 substances that influence the human body, about 60 of which are known carcinogens. Moreover, it is reported that Tobacco smoke affects the normal function of the immunological system, increasing the risk of respiratory infections, which may also contribute to the development of malignancies. During smoking one cigarette, the smoker absorbs about $500 \mathrm{mg}$ of smoke, about $65 \%$ of it is nitrogen and oxygen, and 35\% consists of biologically active substances [1].

The epithelium of the oral mucosa is the first tissue that encounters diverse toxic substances in cigarette smoke [2]. Although smoking induces similar changes in gene expression in the oral and bronchial epithelium concerning carcinogenesis and the activity of selected chemopreventive agents and targeted anticancer drugs, and despite the easy accessibility of the oral epithelial cells-which give insights into tobacco-induced molecular changes in the oral cavity as well as the bronchial epithelium, still the effects of nicotine on epithelial cells of the respiratory tract are well documented [5], but the epithelium of the oral cavity has not been so well explored.

The oral epithelium is a stratified squamous epithelium (keratinized or parakeratinized in areas subjected to mastication as the gingiva and palate). The layers of the oral epithelium represent a continuous process of maturation where the superficial keratinized cells are constantly shed and replaced from below. There is a balance between the dying and proliferating cells. The process of keratinization is considered a physiological cell death, occurring by the mechanisms of apoptosis. However, in apoptosis, cells degenerate into apoptotic bodies, while keratinocytes reach the final stage of differentiation becoming flat, filled with closely packed keratins, and surrounded by a cornified envelope of the mature cell. Apoptosis occurs through two pathways; the extrinsic pathway leading to activation of procaspase 8 
then caspase 3which causes self-destruction of cell, and the intrinsic (mitochondrial) pathway where factors inside the cell can induce apoptosis such as DNA damage, oxidative stress and reactive oxygen species. These factors activate procaspase 9 then caspase 3. Caspase 3 is involved in proteolysis of important proteins and activation of endonucleases that cut DNA into fragments [1].

Caspases are cysteinyl aspartate-specific proteases that play a pivotal role not only in the induction of apoptotic cell death but also in the inflammatory responses against microbial infection. Caspases are divided into three functional groups: apoptosis induction (Caspase-2, $3,6,7,8,9$, and 10), inflammatory responses (Caspase-1, 4, 5, 11, and 12) and differentiation (Caspase-14) [6].

For the previous reasons, this study was designed to study the influences of cigarette smoke on the structure of clinically normal gingival mucosa in healthy men volunteers.

\section{Subjects and methods}

The study was conducted on 18 volunteer male patients who attended the First Dental Center of Egypt (Confi Dent) private clinic in the period from January 2017 till September 2017. This study was approved by the Research and Ethics Committee of Ain Shams University. All 18 participants were fully informed about the objectives of the study and had received printed information and signed a written consent according to the guideline and regulation of the Ethics Committee.

\section{Methodology}

Every patient was subjected to the following:

\section{Careful history taking}

The aim of the study was explained to each subject and if consenting to participate, history was taken, and a questionnaire was filled. History of smoking, alcohol use, medications and systemic diseases was reported. Family history of oral lichen planus and oral cancer in first degree relatives was also reviewed. The level of oral hygiene was established as a function of daily brushing, evaluated as good if the patient brushed three or more times per day, moderate if brushing was once or twice a day, and poor if brushing was infrequent or null. The cause of attending the dental clinic was also reviewed. The significant systemic diseases were hypertension diabetes, hypercholesterolemia, and chronic impairment of renal function and psychiatric status as depression or stress for long period.

For smoking, it was first established if the patient was a smoker or not, and when affirmative, the type and quantity of tobacco smoked per day and the duration of smoking were assessed. It was reported that smokers are those who consumed at least one cigarette per day for a minimum period of one year [7].

\section{The type of consumed cigarette}

The commercial type of cigarette was fixed in all participants. This type contained $1 \mathrm{mg}$ of nicotine and $15 \mathrm{mg}$ of tar in each cigarette.

\section{Examination of the oral cavity}

All participants underwent an oral clinical examination which was performed by experienced stomatologist, through visual inspection and palpation of oral mucosa and teeth. Examination was based on the criteria for the Visual detection of oral mucosal lesions and the World Health Organization (WHO) criteria.

\section{Criteria for patient's selection}

The inclusion criteria: Male volunteers aging from 25-45 years; available at the time of survey; and willing to give written consent were included in this study.

The exclusion criteria: Patients with the following criteria were excluded from the study:

- Patients under medication as corticosteroids or chemotherapeutic drugs.

- Patients diagnosed with cancers, systemic diseases, chronic alcoholism, recent viral infection and drug addiction were also excluded.

- Patients with prosthetic devices (removable or fixed)

- Patients with untreated periodontal diseases.

- Occasional smokers were excluded from the study as well as those who were previously smokers then stopped smoking.

- Pateints with poor oral hygiene.

- Cigar, pipe and shisha smokers were also excluded.

Sample size calculation was kept at minimum required to provide the power sufficient for statistical comparisons.

\section{Study design and grouping}

Patients, who were classified as non-smokers, had no history of tobacco smoking at all. For ethical reasons, smokers were informed about the potentially negative effects of smoking on health and were encouraged to cease smoking.

On observation of any suspicious lesion after examination of the slides, the corresponding patient was informed and advised to be followed up by routine biopsy every 6 months to be referred to oncologist upon need.

Eighteen male participants were included in this study and were classified according to pack year index into three groups:

Group I: non-smokers $(\mathrm{n}=6)$

Group II: pack year index 1-9 $(\mathrm{n}=6)$

Group III: pack year index $>10(\mathrm{n}=6)$

A pack year is a unit for measuring the number of cigarettes a person has smoked over a certain period of time. It is calculated by multiplying the number of packs of cigarettes smoked per day by the number of years of smoking $[8,9]$.

\section{The equation used}

Number of pack years $=($ Number of cigarettes per day/20) $\mathrm{x}$ number of years of smoking ( 1 pack has 20 cigarettes)

For example: a person who has smoked 15 cigarettes a day for 40 years had a $(15 / 20) \times 40=30$ pack-year smoking history.

\section{Sample collection and processing}

Light microscopic study: A $3 \mathrm{~mm}$ punch biopsy was obtained from the outer gingival mucosa behind the right wisdom tooth of the lower 
jaw from each participant. The biopsy was obtained from clinically normal gingival mucous membrane using Kai medical disposable biopsy punch, single use (Kai industries Co. ltd. 1110 Oyana, Seki City, Gifu Pref., 501-39992, Japan) under Mepecaine-L local anesthesia purchased from Alex For Chemical Industries and Drugs. Specimens were immediately fixed in $10 \%$ neutral buffered formalin and were processed into paraffin blocks. Five $\mu \mathrm{m}$ thick sections were cut perpendicular to the epithelium. Then they were stained with $\mathrm{H}$ and $\mathrm{E}$ and Mallory's trichrome stain. Other sections were cut on positively charged slides to be subjected to caspase- 3 immunohistochemical stain [10].

Immunohistochemical study: Caspase-3 (ready to use) was used for detection of apoptosis in the epithelium of gingival mucosa. Paraffin sections of the mucosa and of a positive control (tonsils) were processed. Negative control was processed according to the same protocol, except for the use of the primary antibody [10]. Positive expression of Caspase-3 was detected as brown cytoplasmic reaction with some nuclear staining. The kit was supplied by Lab Vision Corporation. Kato Road, Fremont, USA.

Transmission electron microscopic study: One specimen from each group was immediately fixed in glutaraldehyde and was processed for transmission electron microscopic (TEM) study. Each specimen was cut into small pieces $\left(1 \mathrm{~mm}^{3}\right)$ and rapidly fixed in $2.5 \%$ glutaraldehyde for $24 \mathrm{~h}$. Then they were processed and embedded in Epon resin. Ultrathin sections (50 $\mathrm{nm}$ thickness) were cut and mounted on copper grids [10]. Specimens were examined and photographed using a JEM 1200 EXII, JEOL, Tokyo, Japan transmission electron microscope (TEM) at the Faculty of Science, Ain Shams University.

Morphometric and statistical study: Samples were examined using Leica DM2500 microscope with built in camera (Wetzlar, Germany). All images were digitally acquired using an image analyzer Leica $Q$ win V.3 program (Wetzlar, Germany) installed on a computer in the Histology and Cell Biology department, faculty of Medicine, Ain Shams University. Specimens from all groups were subjected to morphometric study. Measurements were taken from three different slides obtained from each specimen. Five different non-overlapping fields were examined from each slide at objective lens $\times 10$ to measure the first three parameters and $\times 20$ to measure the area percentage:

- Epithelial thickness. It was measured from the surface of the epithelium to the basement membrane by a line perpendicular to the surface.

- Length of epithelial ridges.

- Ratio of basal lamina length to epithelial surface length.

- Area percentage of collagen fibers.

- Area percentage of caspase-3 reaction.

All values were presented as mean \pm SD. The differences among the groups with respect to all measured data parameters were statistically analyzed using one-way analysis of variance and the post-hoc test using SPSS program version 19 (IBM Corporation, Somers, New York, USA). The calculations were considered significant if $\mathrm{P}$ value was less than 0.05 .

\section{Results}

\section{Light microscopic (LM) results}

Examination of $\mathrm{H}$ and $\mathrm{E}$ stained sections from the gingival mucosa (gums) of non-smokers (group I) showed the masticatory mucosa formed of gingival epithelium (stratified squamous non-keratinized and para-keratinized epithelium) and lamina propria. The lamina propria consisted of a papillary layer formed of loose vascular connective tissue and a reticular layer formed of denser connective tissue. Gingival epithelium interdigitated with connective tissue forming interconnected rete ridges that were seen separated by connective tissue papillae (Figure 1A). The gingival epithelium was formed of stratum basale which appeared as a layer of small cuboidal cells resting on the basement membrane. Their nuclei appeared closely packed. Stratum spinosum appeared as many layers of polygonal cells, with central rounded vesicular nuclei. Stratum superficial was seen as flattened superficial cells with flattened nuclei. In the Para keratinized areas, the superficial cells did not lose their nuclei (Figure 1B).

In group II, apparently increased thickness of the epithelium with prominent epithelial ridges was noticed in some areas (Figure 1C). Proliferation of cells of basal layers was noticed. Most cells of stratum spinosum and superficial appeared vacuolated with pyknotic nuclei. Localized areas of proliferation of cells of stratum superficial (epithelial nests) were sometimes noticed (Figure 1D). Intra epithelial lymphocytes, inflammatory cells in the papillary dermis and dividing cells in stratum basale were frequently seen (Figure 1E).

In group III, apparently increased thickness of the epithelium and prominent epithelial ridges were frequently noticed (Figure 1F). Proliferation of cells of stratum basale, vacuolation of cells of stratum spinosum with irregular pyknotic nuclei, karyorrhexis and karyolysis were frequently noticed. Most cells of stratum superficial appeared vacuolated. Some of them appeared with rounded nuclei. Karyorrhexis and karyolysis of most nuclei were seen (Figure 1H). Intra epithelial lymphocytes and mononuclear cellular infiltration in the papillary layer of dermis under the epithelium were also seen (Figure 1G). Increased vascularity of papillary layer of dermis and congestion of blood vessels were also noticed (Figure 1I).

With examination of Mallory's trichrome stained sections in group I, collagen fibers in the papillary layer appeared as fine interlacing bundles. In the reticular layer, collagen fibers were seen arranged in coarse wavy bundles running in different directions. Most bundles were of uniform diameter (Figure 2A and 2B). In group II, irregular short fine collagen fibers were seen in the papillary layer. In the reticular layer, thin irregular disorganized collagen bundles were seen (Figure 2C and 2D). In group III, collagen fibers were seen thin, short disorganized, fragmented and scanty in both the papillary and the reticular layer (Figure $2 \mathrm{E}$ and $2 \mathrm{~F}$ ).

\section{Immunohistochemical results}

Examination of sections from group I showed negative caspase-3 reaction in cells of stratum basale and spinosum. Few cells with positive immune reaction were noticed in stratum superficial (Figure $2 \mathrm{G})$. In group II, caspase- 3 positive immune reaction was seen in the cytoplasm of cells of stratum spinosum and superficial. Negative reaction was seen in cells of stratum basale (Figure $2 \mathrm{H}$ ). In group III, many caspase- 3 positive cells were seen in cells of stratum basale, spinosum and superficial (Figure 2I).

\section{Transmission electron microscopic results}

Examination of ultrathin sections of human gingival mucosa from group I showed the layers of the oral epithelium (stratified squamous epithelium) formed of stratum basale resting on the basement membrane that separated keratinocytes from underlying connective 
tissue (Figure 3A). Keratinocytes of stratum spinosum appeared polygonal in shape with large, central euchromatic nuclei. Desmosomes were seen between the cells (Figure 3B). Cells of the deeper layers of stratum superficial were seen slightly flattened with oval nuclei. Glycogen granules were seen in their cytoplasm (Figure
3C). Superficial cells of stratum superficial were seen more flattened (squamous) with flattened nuclei. Fine micro-ridges were also noticed on the free surface of cells. Desmosomes connected adjacent cell membranes.
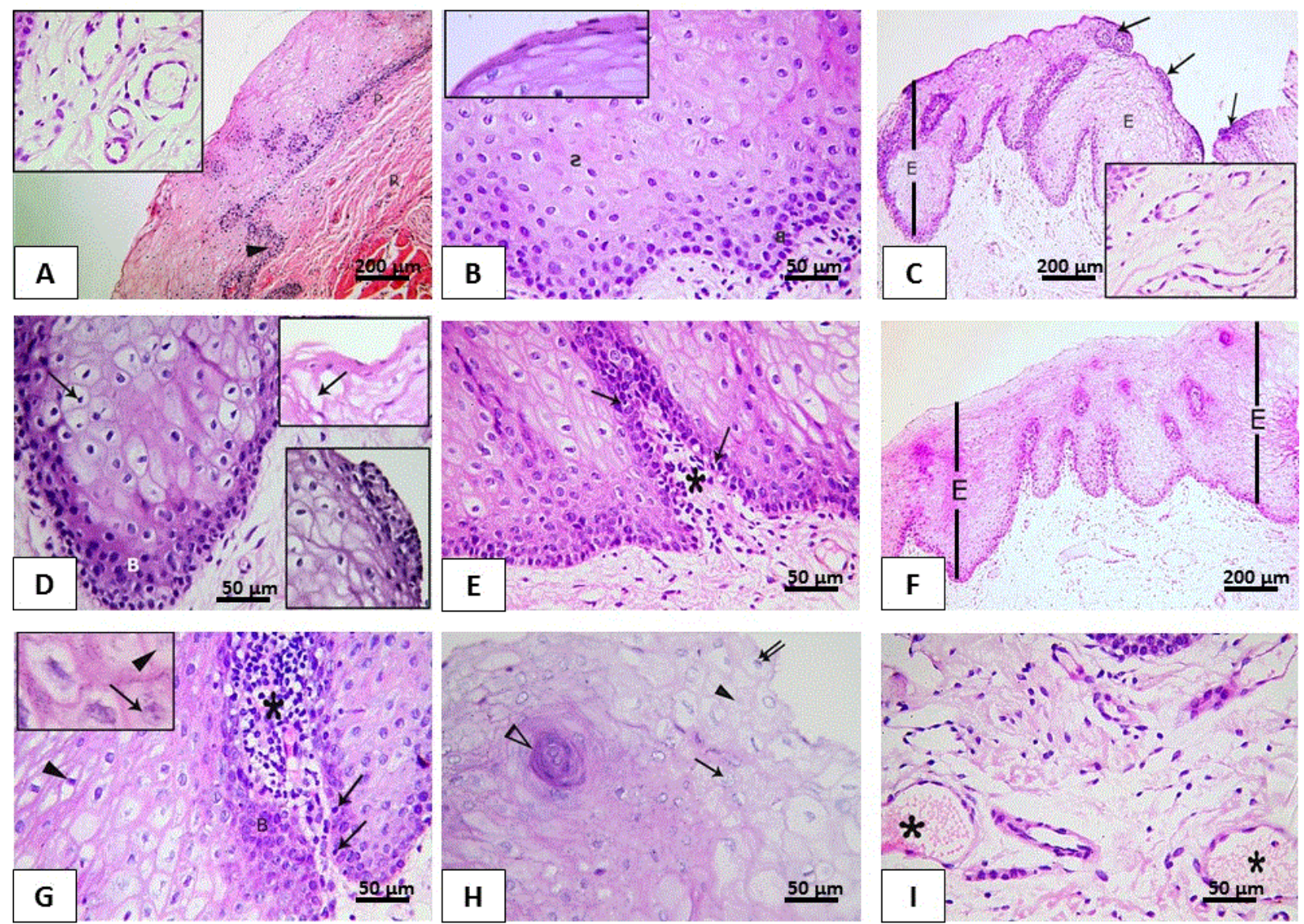

Figure 1: $\mathrm{H}$ and E stained sections. (A and B) Group I: (A) the gingival mucosa is formed of stratified squamous para-keratinized epithelium. The lamina propria consists of papillary layer $(\mathrm{P})$ formed of loose connective tissue. The reticular layer (R) is formed of denser connective tissue. Connective tissue papillae interdigitate with epithelial ridges (pegs) () Inset: blood vessels in the papillary layer. (B) The gingival epithelium is formed of stratum basale (B) which is a layer of small cuboidal cells resting on the basement membrane. The nuclei appear closely packed. Stratum spinosum (S) appears as many layers of polygonal cells with central rounded nuclei. Inset: stratum superficial in the para-keratinized area appears as flattened superficial cells with flattened nuclei. (C-E) Group II: (C) Area of apparent increase thickness of the epithelium (E), prominent epithelial ridges, numerous dermal papillae and areas of localized cellular aggregation at the surface $(\uparrow)$ are seen. Inset: blood vessels in the papillary layer. (D) area of proliferation of basal layer (B) is noticed. Most cells of stratum spinosum and are seen vacuolated with pyknotic nuclei $(\uparrow)$. Upper inset: superficial vacuolated cells $(\uparrow)$ are seen in the para-keratinized epithelium. Lower inset: localized area of proliferation of cells of stratum superficial (epithelial nest). (E) showing intra epithelial lymphocytes ( $\uparrow$ ) and inflammatory cells in the papillary dermis $\left(^{*}\right)$. (F-I) Group III: (F) Areas of apparent increase in thickness of the epithelium (E) and prominent epithelial ridges can be seen. (G) Proliferation of cells of stratum basale (B), vacuolation of cells of stratum spinosum with irregular pyknotic nuclei () and intra-epithelial lymphocytes $(\uparrow)$ are seen. Mononuclear cellular infiltration $\left({ }^{*}\right)$ can also be seen in the papillary layer of dermis under the epithelium. Inset: karyorrhexis $(\uparrow)$ and karyolysis () of nuclei of cells of stratum spinosum. (H) Most cells of stratum superficial are vacuolated. Karyorrhexis $(\uparrow)$ and karyolysis () of some nuclei are seen. Some superficial cells show rounded nuclei $(\uparrow \uparrow)$. Nest of squamous epithelium is seen $(\Delta)$. (I) increase vascularity of papillary layer of dermis and congestion ${ }^{*}$ ) of some vessels can be seen. A, C and F (X 100); B, D, E, G, H and I (X 400); Insets of A, B, C and D (X 400); Inset of G (X 1000). 

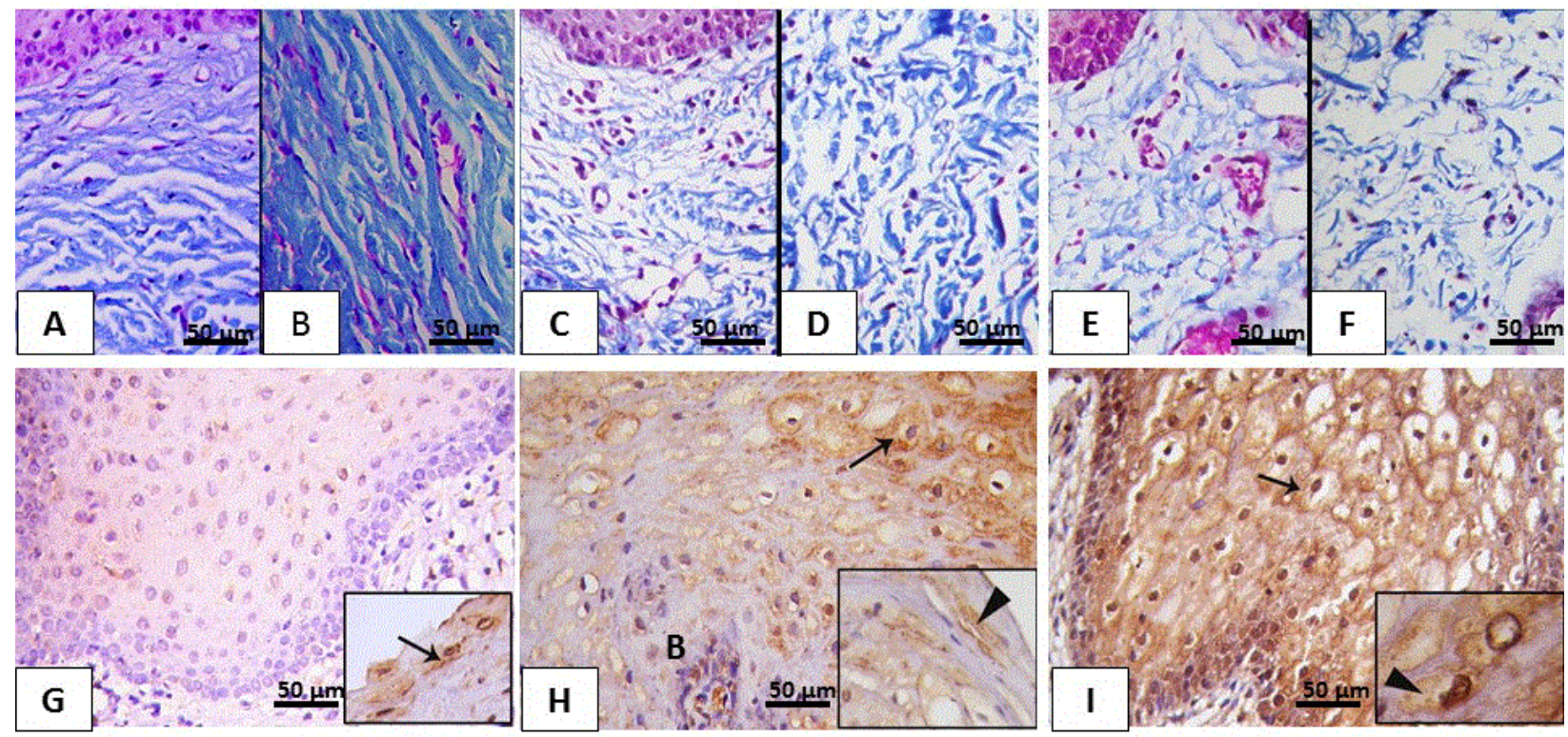

Figure 2: (A-F) Mallory stained section. (A and B) Group I: (A) Collagen fibers in the papillary layers appear as fine interlacing bundles. (B) In the reticular layer, collagen fibers are arranged in coarse, wavy bundles running in different directions. Most bundles are of uniform diameter. (C and D) Group II: (C) showing irregular short fine collagen fibers in the papillary layer. (D) In the reticular layer thin irregular disorganized collagen bundles are seen. (E and F) Group III: (E) Showing thin short and scanty collagen fibers in the papillary layer. (F) In the reticular layer, thin disorganized fragmented and scanty collagen fibers are seen. (G-I) Caspse-3 immune reaction. (G) Group I: negative caspase-3 reaction is seen in cells of stratum basale and spinosum. Inset: positive immune reaction in cells of stratum superficial ( $\uparrow$ ). (H) Group II: negative caspase- 3 reaction is seen in cells of stratum basale (B) and positive reaction is seen in the cytoplasm of cells $(\uparrow)$ of stratum spinosum. Inset: positive immune reaction in cells of stratum superficial (). (I) Group III: many caspase-3 positive cells ( $\uparrow$ ) are seen in cells of stratum basale and spinosum. Inset: caspase-3 positive reactions are seen in cells () of stratum superficial. A-F (Mallory`s trichrome stain X 400); G- I (Caspase-3 × 40); Insets of G, H and I × 400 .

Detached cells were sometimes noticed (Figure 3D). In group II, cells of stratum basale were seen with euchromatic nuclei and resting on basement membrane separating them from underlying connective tissue. Desmosomes were seen between adjacent cells (Figure 4A). Most cells of stratum spinosum appeared with intracellular vacuoles (Figure 4B), disruption of desmosomal plaques and widening of the intercellular spaces. Apoptotic cells were also seen containing no nuclei (Figure 4C).

The deep layers of stratum superficial showed large amount of intracellular electron dense glycogen granules (Figure 4D). Superficial cells of stratum superficial were seen flattened with fine micro-ridges on their free surface. Some nuclei appeared with disturbed unclear membrane and chromatin arrangement (Figure 4E). In group III, cells of stratum basale were seen resting on irregular basement membrane.

Electron dense ellipsoid granules were also seen. Wide intercellular spaces and faint nuclei were frequently noticed (Figure 5A). Intracellular electron dense glycogen granules, wide intercellular space and disruptions of desmosomal attachment plaques were frequently seen (Figure 5B). Irregular nuclear envelope, clumped chromatin and perinuclear glycogen granules were seen in most cells of stratum superficial. Disturbed micro-ridges were also noticed on the free surface of the squamous cells (Figure 5C). 
Citation:Hamam GG, El-Waseef DAEA (2018) Effect of Cigarette Smoking on Human Gingival Mucosa-Histological and Morphometric Study. J Cytol Histol 9: 517. doi:10.4172/2157-7099.1000517
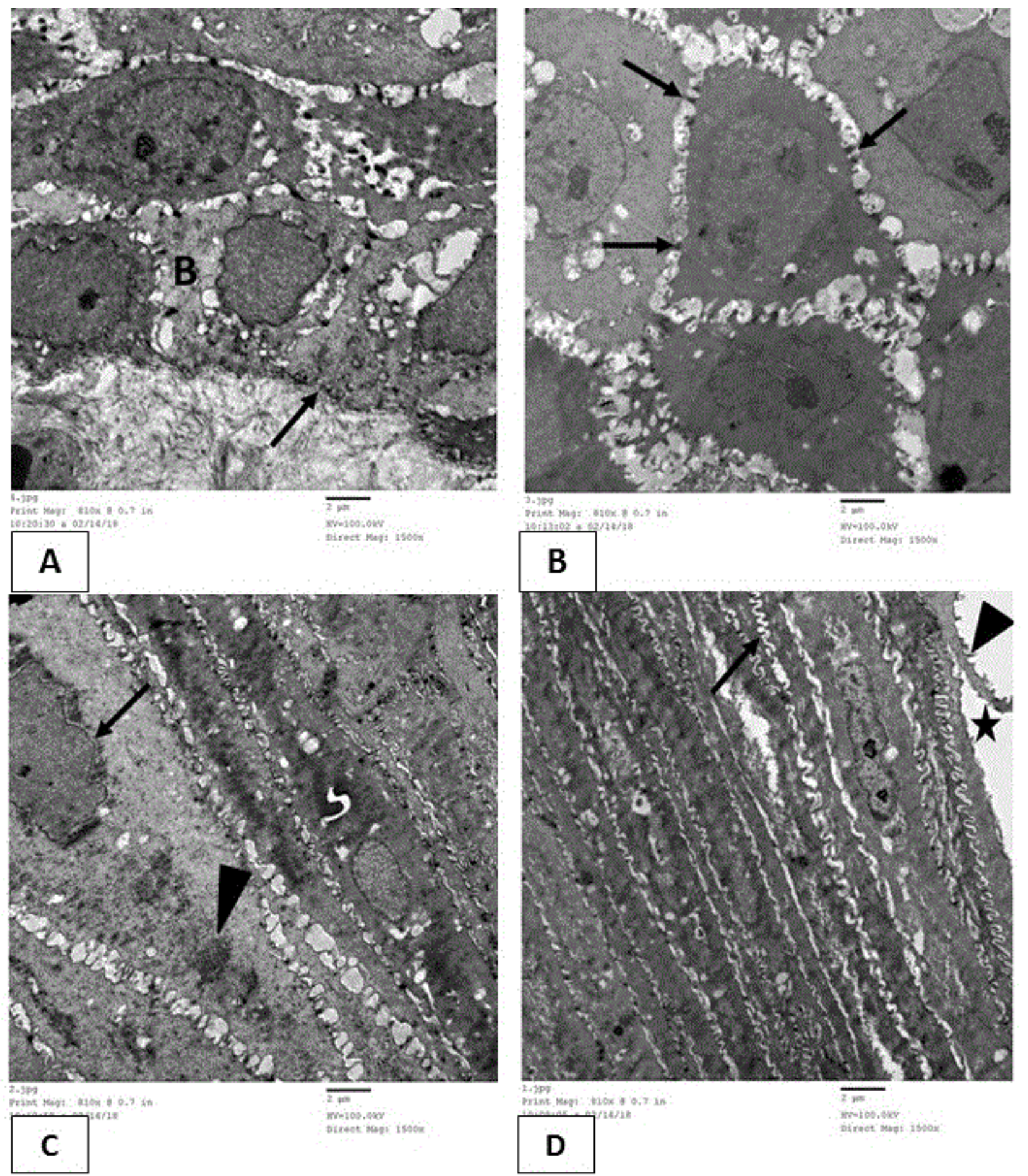

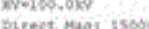

Figure 3: (A-D) Group I: (A) Stratified squamous lining of the human gingival mucosa. A continuous basement membrane ( $\uparrow)$ separates keratinocytes in the stratum basale (B) from underlying connective tissue. (B) Keratinocytes in the stratum spinosum are polygonal in shape with large, central euchromatic nuclei with two nucleoli. Desmosomes ( $\uparrow$ ) are seen between the cells. (C) Cells of the deeper layers of stratum superficial are seen slightly flattened with euchromatic nuclei $(\uparrow)$ and glycogen granules () in their cytoplasm. (D) Superficial cells of stratum superficial are more flattened (squamous) with flattened nuclei. Fine micro-ridges are seen on the free surface of the squamous cells (). Desmosomes connect adjacent cell membranes $(\uparrow)$. Beginning of detachment of cells is seen $\left.{ }^{*}\right)$. A, B, C and D $($ TEM $\times 1500)$. 


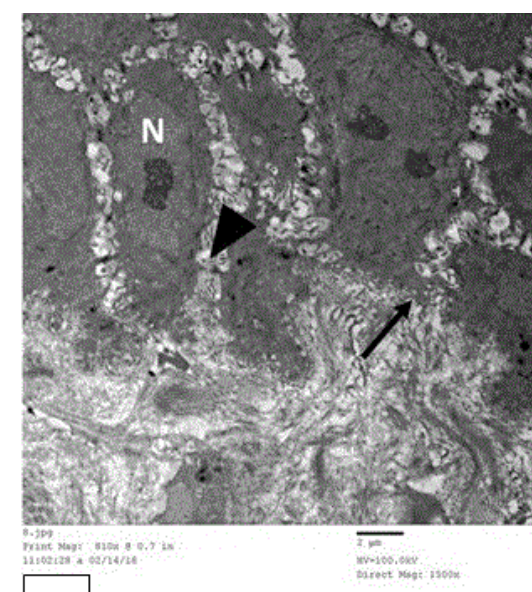

A

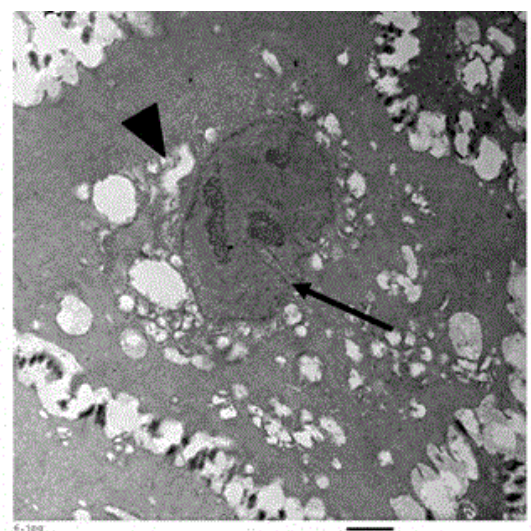
B
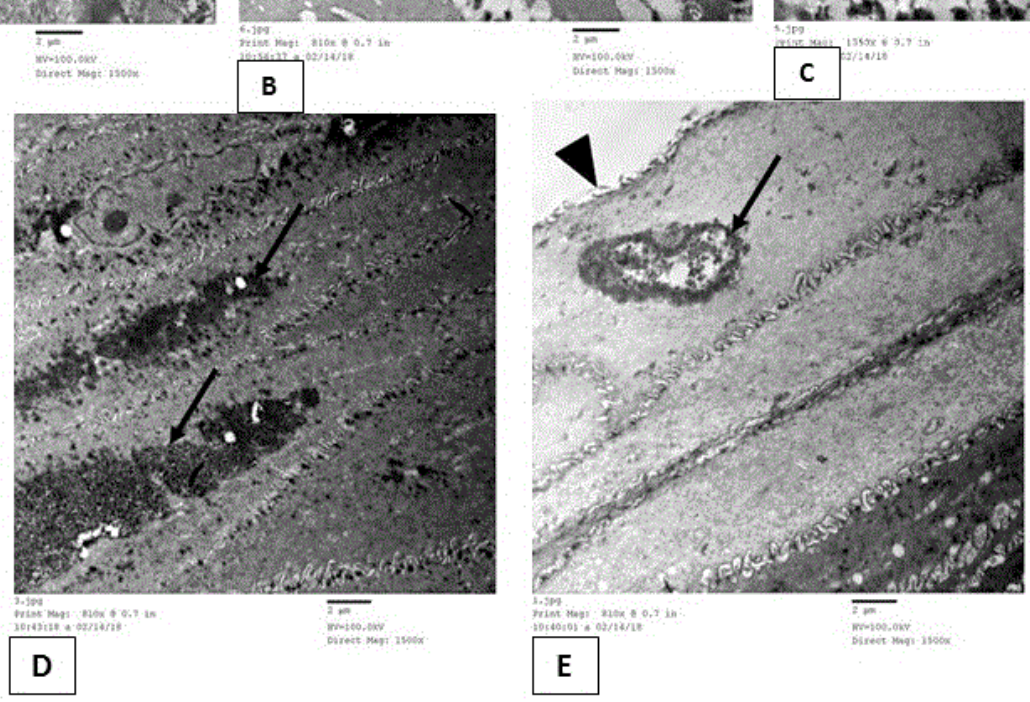

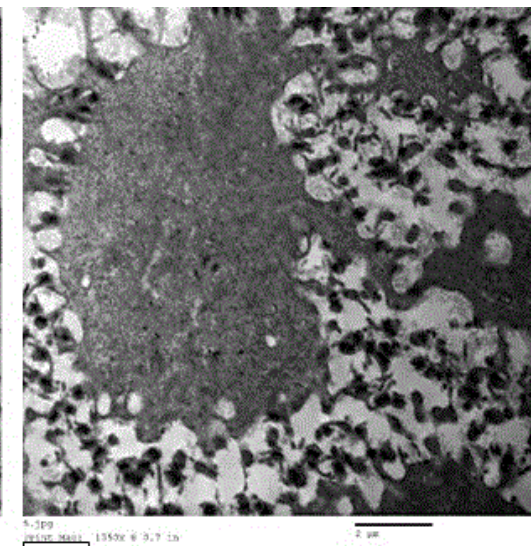

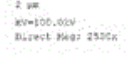

Figure 4: (A-E) Group II: (A) cells of stratum basale are seen with euchromatic nuclei (N). irregular Basement membrane ( $\uparrow$ ) separates them from underlying connective tissue. Desmosomes () are seen between adjacent cells. (B) indented nucleus ( $\uparrow$ ) and intracellular vacuoles () are seen in cells of stratum spinosum. (C) apoptotic cell, disruption of desmosomal plaques and wide intercellular spaces are seen between cells of stratum spinosum. (D) The deep layers of stratum superficial show large amount of intracellular electron dense glycogen granules ( $\uparrow$ ). (E) Superficial cells of stratum superficial are seen flattened with fine micro-ridges on their free surface (). A nucleus ( $\uparrow$ ) with disturbed nuclear membrane and chromatin arrangement is seen. A, B, D and E (TEM × 1500); C (TEM X 2500).

\section{Histomorphometric results}

In the present study, 18 individuals were included. The age range of non-smokers was $28-42$ years with a mean of $32.8 \pm 6.2$ years; the age range of smokers in group II was 26-38 years with a mean of $32 \pm 4.5$ years and the age range of smokers in group III was $28-45$ years with a mean of $36.3 \pm 11.1$ years (Table 1 ).

In group II, the range of daily cigarette consumption was 5-15 cigarettes $(9.6 \pm 7.4)$, for $4-15$ years $(9.2 \pm 4.1)$ with range of pack year index 1-7.5 (4.5 \pm 2.8$)$. In group III, the range of daily cigarette consumption was $12-30(20.3 \pm 9.5)$ for $10-30$ years $(19 \pm 10)$ with range of pack year index 10-30 (16.5 \pm 11.3$)$ (Table 1).

The mean thickness of both the epithelium and the epithelial ridges were significantly increased in groups II and III compared to control group. The percentage ratio of basal lamina length to epithelial surface length was significantly increased in groups II and III compared to control group. These parameters showed no significant difference between groups II and III (Table 2 and Figure 6). 


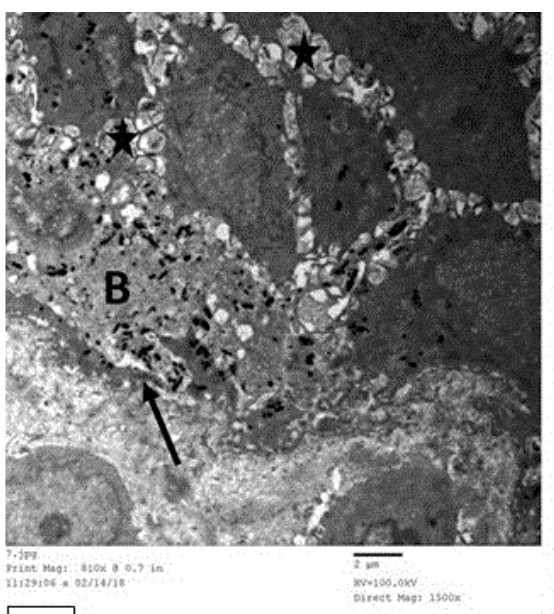

A

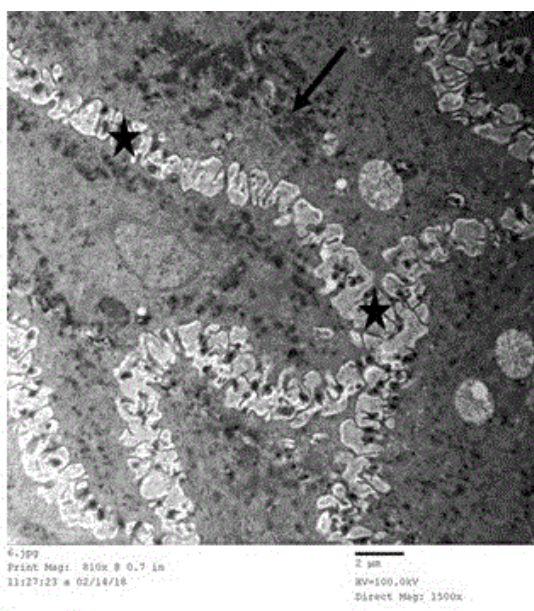

B

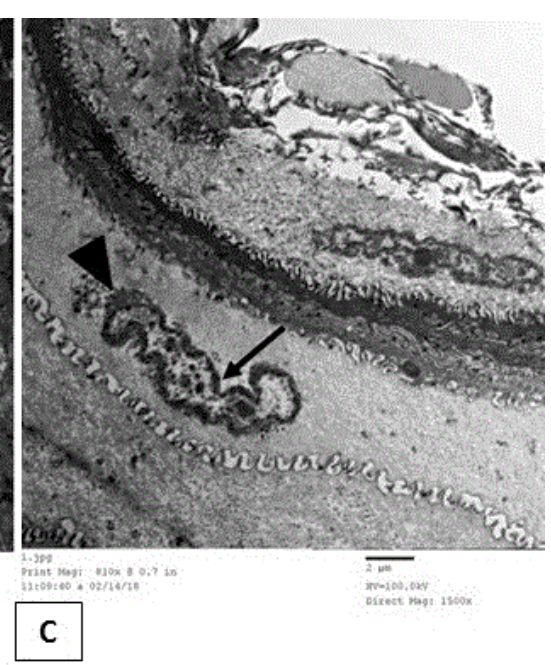

Figure 5: (A-C) Group III: (A) Irregular basement membrane $(\uparrow)$ separates keratinocytes in the stratum basal (B) from underlying connective tissue. Cells are seen with electron dense melanin granules. Wide intercellular spaces ${ }^{*}$ ) and faint nuclei are seen. (B) intracellular electron dense glycogen granules, wide intercellular space $\left({ }^{\star}\right)$, disruptions of desmosomal attachment plaques between cells of stratum spinosum are seen. (C) Stratum superficial is seen as flattened cells with flattened nuclei. Irregular nuclear envelope $(\uparrow)$, clumped chromatin and perinuclear glycogen granules () are seen. Disturbed micro-ridges can be seen on the free surface of the squamous cells (). A, B and C (TEM $\times 1500)$.

The mean area percentage of collagen fibers in the dermis was significantly decreased in groups II and III compared to control group. Group III also showed significant decrease compared to group II (Table 2 and Figure 6).

There was a significant increase in the mean area percentage of caspase- 3 reaction in the epidermis in groups II and III compared to control group. A significant increase was also noticed in group III compared to group II (Table 2 and Figure 6).

\begin{tabular}{|l|l|l|l|}
\hline & Group I & Group II & Group III \\
\hline Number of cases & 6 & 6 & 6 \\
\hline Age & $32.8 \pm 6.2$ & $32 \pm 4.5$ & $36.3 \pm 11.1$ \\
\hline Daily consumption & - & $9.6 \pm 7.4$ & $20.3 \pm 9.5$ \\
\hline Duration-year & - & $9.2 \pm 4.1$ & $19 \pm 10$ \\
\hline Pack Year Index & 0 & $4.5 \pm 2.8$ & $16.5 \pm 11.3$ \\
\hline
\end{tabular}

Table 1: History and mean \pm SD of smoking habits characteristics in different groups.

\begin{tabular}{|l|l|l|l|}
\hline & Group I & Group II & Group III \\
\hline Epithelial thickness $(\mu \mathrm{m})$ & $310.78 \pm 10.9$ & $401.76 \pm 16.7$ & $441.10 \pm 23.3$ \\
\hline Length of epithelial ridges $(\mu \mathrm{m})$ & $95.75 \pm 5.2$ & $180.48 \pm 7.7$ & $208.16 \pm 8.4$ \\
\hline Ratio of Basal lamina length/epithelial surface length $(\%)$ & $142.87 \pm 4.3$ & $201.1 \pm 6.7$ & $211.31 \pm 7.1$ \\
\hline Area\% of collagen fibers & $42.03 \pm 1.2$ & $34.4 \pm 2.3^{*}$ & $28.11 \pm 0.8^{*}$ \\
\hline Area\% of caspase-3 & $6.46 \pm 1.0$ & $22.9 \pm 2.3^{*}$ & $35.43 \pm 3.1^{*}$ \\
\hline $\begin{array}{l}\text { Significant increase compared to control } \\
\text { "Significant change compared to other groups }\end{array}$ & & & \\
\hline
\end{tabular}

Table 2: Mean \pm SD of epithelial thickness, length of epithelial ridges, basal lamina/epithelial surface length, area\% of collagen fibers and area $\%$ of caspase-3 positive immune reaction in different groups. 


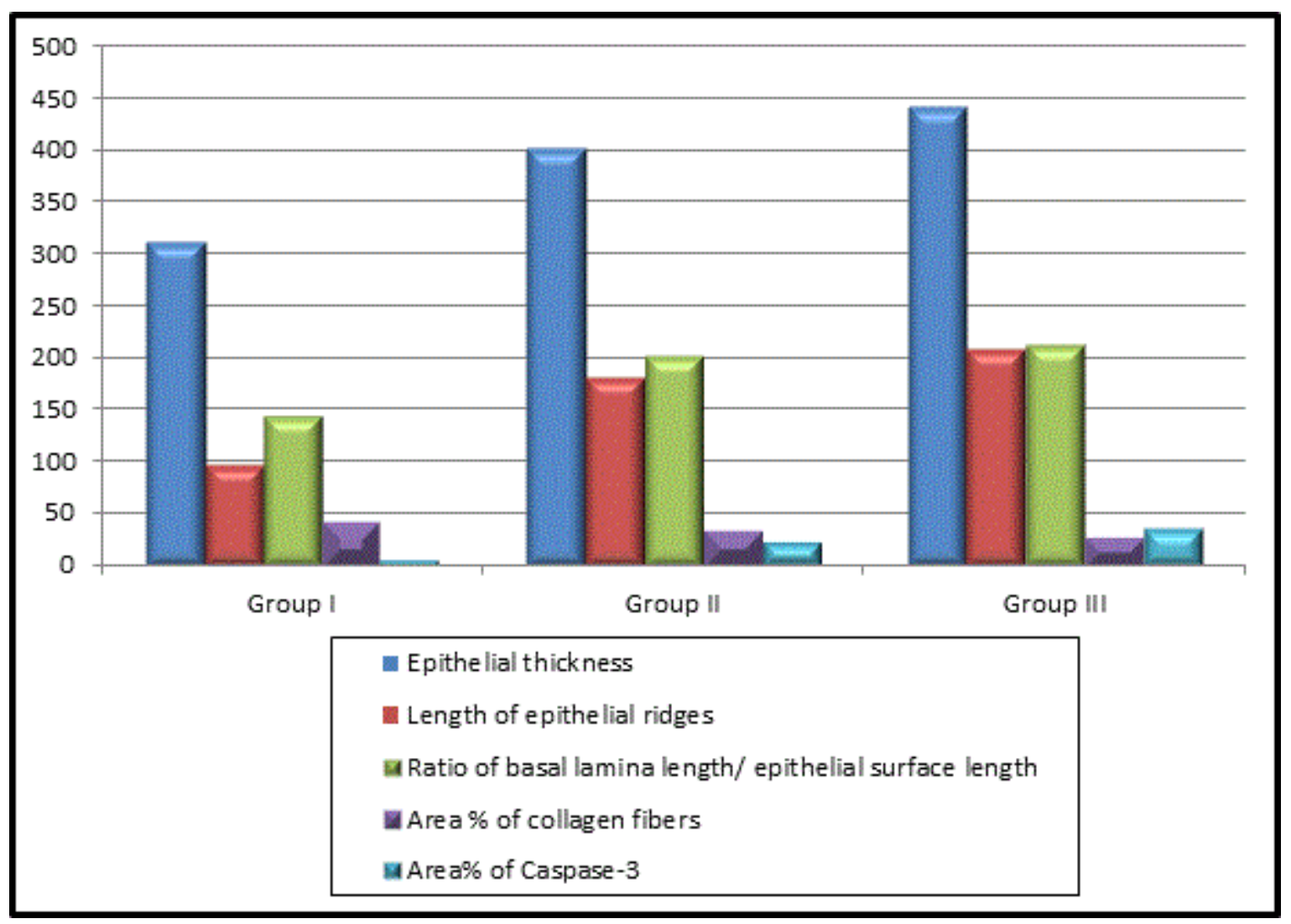

Figure 6: The mean thickness of epithelium, the mean thickness of epithelial ridges, the ratio of basal lamina length/epithelial surface length, the mean area percentage of collagen fibers in dermis and Caspase- 3 reaction in different groups.

\section{Discussion}

The first exposure of cigarette smoke is experienced by the oral tissues, and its chemical components are rapidly absorbed and circulate in the blood stream [11]. So, we sought to investigate the effects of whole cigarette smoke on the structure of normal human gingival mucosa in healthy males. Women were not included in this study, to avoid cellular changes that occur during menstruation, after menopause and in pregnancy, as previously reported by some authors [12].

In the current study, pack year index was used to evaluate the effects of smoking as it was reported that pack year index describes the degree of exposure to tobacco [8].

In the current study, basal layer hyperplasia was noticed in groups II and III and the mean thickness of gingival epithelium was significantly increased in both groups, compared to control group. This was explained by some authors who reported that cigarette smoke directly activates epithelial cell proliferation $[13,14]$ and causes basal layer hyperplasia in the mucosa of pharynx, larynx and tongue of rats [13]. Proliferation of basal cell layer of the epithelium might be a protective mechanism against irritation of smoking which leads to proliferative response of basal stem cells.

In the current study, elongation of epithelial ridges and increase ratio of basal lamina length to epithelial surface length were noticed in groups II and III. It was reported that the length of the basal lamina is directly related to the overall number of basal cells that participate in epithelial renewal. The longer the basal lamina below the epithelial surface; the greater is the number of the basal cells that can enter mitosis [15]. It was also reported that tobacco components stimulate production of proinflammatory cytokines, which could induce epithelial cells proliferation and migration to help epithelization during wound healing [16]. Also, it was previously reported that nicotine, a component of tobacco smoke, significantly stimulates cell growth [17]. These facts could be a good explanation for the thickening of epithelium observed in groups II and III in the current study.

In the current study, localized areas of epithelial hyperplasia (cell nest) were noticed in groups II and III. It was reported that remnant of dental lamella is called small nests of squamous epithelium. These epithelial nests may proliferate and undergo degeneration forming gingival cyst, which is premalignant [18]. Metaplasia represents an adoptive and reversible response to persistent injury of the epithelium caused by chronic infections. It results from a reprogramming of epithelial stem cells that begin to differentiate into new cell lineage [19].

Cigarette components as nicotine, carbon monoxide, and reactive oxygen species can penetrate the cells and induce DNA damage, leading to various mutations [16]. In groups II and III, various changes were noticed in the nuclear structure ranging from irregular nuclear membrane, clumped chromatin, pyknosis, karyolysis and karyorrhexis. It was reported that nuclear damage triggers impairment of p53/p21 
pathways. Stem cells need regulated amount of p21which is a cell cycle inhibitor. It has an essential role in maintaining stem cells quiescence. In the absence of $\mathrm{p} 21$, stem cells fail to maintain quiescence resulting in hyperproliferation and expansion of progenitor cell pools resulting in exhaustion of stem cell pool [20].

It was reported that normal renewal of the oral epithelium is the result of equilibrium between rate of proliferation and rate of cell death [21]. Very little is known about the possible impact of tobacco smoke on the apoptosis of epithelial cells in the oral cavity, and observations vary, from no changes on the level of apoptosis to increased levels of hypodiploid cells in the epithelium of smokers through the inhibition of apoptosis under influence of nicotine. However, these observations are based on in vitro cultured keratinocytes and animal models [1]. In the current study, a significant increase in the mean area percentage of caspase-3 expression was noticed in smokers of groups II and III, meaning that smoking increased the rate of cell death. However, our light microscopic and morphometric results showed a significant increase in the thickness of the epithelium and in the proliferation of the basal layer in groups II and III. The previous findings mean that the proliferative capacity of the epithelium under the effect of smoking markedly exceeded the rate of cell death. Added to the effect of carcinogenic products in cigarette smoke, all these factors put the gingival epithelium at a high risk of developing cancers. It was also reported that apoptosis progression relies on the interaction between pro-survival factors and pro-apoptotic factors, and this balance decides whether a cell under stress lives or dies. Pro-survival proteins can inhibit the apoptotic cytoplasmic caspases. Injury to superficial airway epithelium is normally accompanied by mitotic activity in the remaining cells and rapid restoration of the denuded surface. Tobacco smoke is a potent stimulus of DNA damage by oxidant injury. Tobacco smoke may affect the expression and activity of proteins associated with cell proliferation and cell apoptosis thus altering physiological reparative mechanisms [22].

An important property of epithelium is its ability to function as a barrier. Desmosomes or maculae adherens are modifications of adjacent membranes of cells to ensure proper barrier [23]. In the current study, ultra-structural analysis of gingiva of groups II and III showed disruption of desmosomes between cells of stratum spinosum. This might be explained by the fact that cigarette smoke increases cytokines which destroy the cell-cell interaction proteins (e.g. Ecadherin) [16].

In electron microscopic examination of groups II and III, intracellular glycogen was noticed in cells of stratum spinosum and superficial of the gingival epithelium. This might explain the vacuolation seen in $\mathrm{H}$ and $\mathrm{E}$ stained sections in the epithelium of these two groups.

Ellipsoid electron dense granules mostly melanin granules were noticed in cells of stratum basale in group III. Other authors also reported increased melanin granules in smokers. They attributed this to the development of smokers' melanosis [24].

Intraepithelial lymphocytes were noticed in groups II and III. It was reported that intraepithelial lymphocytes are found in epithelial mucosal linings. Upon encountering antigens, they immediately release cytokines and cause killing of infected target cells. They act at epithelial border to eliminate injured and infected cells. It was reported that in mucosal cell surface, T cell receptors are mainly of $\gamma$ and $\delta$ chains, not $\alpha$ and $\beta$ chains. The $\gamma / \delta$ T cells are characterized by being a bridge between the innate and adaptive immune system. They act as the first line of defense against microbes [25].

In the current study, inflammatory cells were noticed in lamina propria of groups II and III. Similar finding was also noticed by some authors [26]. It was reported that tobacco components produce proinflammatory cytokines that cause chronic inflammation. Cytokines are inflammatory factors that are normally secreted due to tissue injury to promote repair process. Till now, the cytokines that have been found to increase after exposure to cigarette smoke are the interleukins (IL)-1, IL-6, IL-8, IL-10, tumor necrosis factor- $\alpha$, as well as transforming growth factor- $\beta$, granulocyte-macrophage colonystimulating factor and monocyte chemoattractant Protein. The role of the inflammatory cytokines is to recruit the immune cells during the infection [27].

Tobacco smoke contains nicotine which is a cytotoxic substance that induces immune-suppression, defective neutrophil functions and impaired gingival health [28]. It was reported that cigarette smoke suppresses the activation of epithelial-mediated innate immune responses to bacterial infection.

The epithelium is a non-vascular tissue that depends on the adjacent connective tissue. Inflammation modifies the blood availability and impedes the elimination of metabolites from the epithelium [29]. In the current study, numerous capillaries and congestion of blood vessels were noticed in group III. This was in accordance with some authors $[13,26]$. On the contrary, it was reported that moderate negative correlation was found between pack years and the number of blood vessels [30]. It was also reported that decrease in capillary diameter and density of blood vessels were noticed in gingival tissues of smokers. This is sometimes confused with gingival health status as noticed by reduction of tissue redness and bleeding upon probing [13].

In the current study, Mallory`s triple stain revealed disorganization of collagen fibers and significant decrease in mean area percentage of collagen fibers in group III compared to control group. This was explained by the fact that cigarette smoke strongly increases cytokines which induce production of protease enzymes, including matrix metalloproteinases (MMPs), elastase, and gelatinase, which destroy the extracellular matrix proteins (e.g. collagen, elastin, and gelatin) and cell-cell interaction proteins (e.g. E-cadherin) during the infection or tissue repair. It was also reported that inflammation causes connective tissue disorganization [29].

Human gingival fibroblasts rapidly uptake and accumulate high levels of nicotine in vitro, most of which remains inside the fibroblasts where it can affect cell metabolism. In addition, nicotine inhibits proliferation and migration of human periodontal ligament fibroblasts. Nicotine also acts on lysosomes by lowering the inner $\mathrm{pH}$ or by enlarging them [31]. Others reported that normal function of gingival fibroblasts is essential for maintenance of the gingival extracellular matrix, but under inflammation of the gingiva -which may occur with tobacco use-, they can also act in its destruction. Effect of tobacco on gingival fibroblast proliferation, production of fibronectin, production and breakdown of type I collagen, was studied by some authors. They found that nicotine at $\geq 0.025 \%$ significantly increased collagenase activity in vitro; inhibited growth of gingival fibroblasts and their production of fibronectin and collagen and promoted collagen breakdown. This suggested that nicotine itself might augment the destruction of the gingival extracellular matrix [32].

On the contrary, greater collagenization was noticed in connective tissue of smokers [33]. Clinically, it has been widely recognized that 
fibrosis is frequently observed in the smoker's gingiva. Nicotine increased the production of connective tissue growth factor protein in gingival fibroblasts and promoted periodontal fibrosis [29]. This discrepancy might be attributed to the fact that the effect of nicotine on fibroblast viability and growth, depends on the concentration as well as duration of cell exposure [29].

In the current study, group III showed significant increase in the mean area parentage of caspase- 3 positive reaction in epithelial cells and a significant decrease in the mean area percentage of collagen fibers in the lamina propria, compared to group II. It was reported that the amount and the duration of exposure to a carcinogen are often combined to give a single measure of lifetime cumulative dose [34].

There are controversial observations on the effect of smoking on gingival tissue. Some reports indicated that smokers had more gingival inflammation; others observed less gingival inflammation in smokers than non-smokers. In this study inflammation was more often seen in smokers. When collagen fibers were evaluated, we have also found that they decreased in lamina propria of smoker's gingiva. Others reported increase collagen fibers. This discrepancy could be attributed to smaller number of samples in this study, as well as the fact that our samples were obtained from the gingiva of the lower jaw and it was reported that the impact of cigarette smoke is greater on the upper jaw compared to the lower as they were more exposed to tobacco smoke [33].

\section{Conclusion}

The present study indicated that despite the normal appearance of gingiva in smokers, cigarette smoke could change the structure of human gingival mucosa in a dose dependent manner.

\section{Recommendations}

A gingival biopsy every 6 month is recommended for Tobacco smokers as a follow up, not only for early detection of any oral epithelial neoplasms, but also as a detector for the respiratory epithelial neoplasms which are more dangerous and more difficult to be biopsied.

Tobacco smoking is a Public Health problem with serious side effects and it needs intensive and comprehensive tobacco control efforts.

\section{Acknowledgements}

We would like to thank Professor Dr. Hussein M. Taher and his team for their kind helps in history and consent taking, in examination and obtaining the specimens.

\section{Ethical Approval}

All procedures performed in studies involving human participants were in accordance with the ethical standards of the institutional and national research committee.

\section{References}

1. Michcik A, Cichorek M, Daca A, Chomik P, Wojcik S, et al. (2014) Tobacco smoking alters the number of oral epithelial cells with apoptotic features. Folia Histochem Cytobiol 52: 60-68.
2. Qian YJ, Wang X, Gao YF, Duan N, Huang XF, et al. (2015) Cigarette smoke modeulates NOD1 signal Pathway and Human $\beta$ Defensins Expression in Human Oral Mucosa. Cell Physiol Biochem 36: 457-473.

3. Mr P, Guruprasad Y, Jose M, Saxena K, Prabhu V (2014) Comparative Study of Genotoxicity in Different Tobacco Related Habits using Micronucleus Assay in Exfoliated Buccal Epithelial Cells. J Clin Diagn Res 8: ZC21-ZC24.

4. Villa A, Gohel A (2014) Oral potentially malignant disorders in a large dental population. J Appl Oral Sci 22: 473-476.

5. Sridhar S, Schembril F, Zeskind J Shah V, Gustafson AM, Steiling K, et al. (2008) Smoking-induced gene expression changes in the bronchial airway are reflected in nasal and buccal epithelium. BMC Genomics 9: 259.

6. Uchiyama R, Tsutsui H (2015) Caspases as the key effectors of inflammatory responses against bacterial infection. Arch Immunol Ther Exp (Warsz) 63: 1-13.

7. Zarate AM, Brezzo MM, Secchi DG, Barra JL, Brunotto M (2013) Malignancy risk models for oral lesions. Med Oral Patol Oral Cir Bucal 18: e759-e765.

8. Indrayan A, Kumar R, Dwivedi S (2008) A simple index of smoking. COBRA Preprint series.

9. National Cancer Institute definition of pack year.

10. Suvarna K, Layton C, Bancroft J (2013) Theory and practice of histological techniques (7th edn.). Churchill Livingston, USA.

11. Ng TK, Huang L, Cao D, Yip YW, Tsang WM, et al. (2015) Cigarette smoking hinders human periodontal ligament-derived stem cell proliferation, migration and differentiation potentials. Sci Rep 5: 7828.

12. Hashemipour MA, Aghababaie M, Mirshekari TR, Asadi-Shekaari M, Tahmasbi-Arashlow M, et al. (2013) Exfoliative cytology of oral mucosa among smokers, opium addicts and non-smokers: A cytomorphometric study. Arch Iran Med 16: 725-730.

13. Jalayer Naderi N, Semyari H, Elahinia Z (2015) The impact of smoking on gingival: A histopathological study. Iran J Pathol 10: 214-220.

14. Wang X, Qian YJ, Zhou Q, Ye P, Duan N, et al. (2014) Caspase-12 silencing attenuates inhibitory effects of cigarette smoke extract on NOD1 signaling and hBDs expression in human oral mucosal epithelial cells. PLoS One 9: e115053.

15. Sanja TS, Jovan C, Tatjana K, Zoran M (2016) Differential histomorphometric changes in normal and inflamed gingival epithelium. Sanamed 11: 197-201.

16. Alharbi IA, Rouabhia M (2016) Repeated exposure to whole cigratte smoke promotes primary human gingival epithelial cell growth and modulates keratin expression. J Periodont Res 51: 630-638.

17. Tarakji B, Kujan O, Nassani MZ (2010) An immunohistocehmical study of the distribution of p16 protein in oral mucosa in smokers, nonsmokers and in frictional keratosis. Med Oral Patol Oral Cir Bucal 15: e681-e684.

18. Mills SE (2012) Histology for pathologists (4thedn), Lippincoti Williams and Wilkins, Wolters Kluwer. Philadelphia, USA, pp: 433-478.

19. Ross MH, Pawlina W (2015) Histology: A text and atlas with correlated cell and molecular biology (7th edition). Wolters Kluwer Philadelphia, New York, p. 858.

20. Herbig U, Jobling WA, Chen BP, Chen DJ, Sedivy JM (2004) Telomere shortening triggers senescence of human cells through a pathway involving ATM, p53 and p21 (CIP1) but not p16 (INK4a). Mol cell 21: 501-513.

21. Sell S (2013) Stem cells handbook (2nd edition). Humana Press, p. 229.

22. Chiappara G, Giomarkaj M, Virzi A, Sciarrino S, Ferraro M, et al. (2013) The role of p21 Waf1/Cip1 in large airway epithelium in smookers with and without COPD. Biochim Biophys Acta 1832: 1473-1481.

23. Nanci A (2008) Ten cate's oral histology development, structure, and function. Mosby Inc. of Elseivier Inc. International, p: 373.

24. Al-Attas SA, Ibrahim SS, Amer HA, Darwish Zel-S, Hassan MH (2014) Prevalence of Potentially Malignant Oral Mucosal Lesions among Tobacco Users in Jeddah, Saudi Arabia. Asian Pac J Cancer Prev 15: 757-762. 
25. Paul WE (2013) Fundamental immunology (7thedn), Lippincott Williams and Wilkins, Philadelphia, USA.

26. Rahman BU, Rahman MM, Arslan A (1994) The effects of cigarette smoking on human gingival tissues (a histopathological study). J Pak Med Assoc 44: 210-212.

27. Mokeem SA, Vellappally S, Preethanath RS, Hashem MI, Al-Kheraif AA et al. (2014) Influence of smoking on clinical parameters and gingival crevicular fluid volume in patients with chronic periodontitis. Oral Health Dent Manag 13: 469-473.

28. Ashraf T, Jan SM, Behal R (2017) Evaluation of effects of smoking on gingival thickness-A clinical study. IAIM 4: 182-186.

29. Villar CC, de Lima AF. Smoking influence 42 in paper 2.

30. Kumar V, Faizuddin M (2011) Effect of smoking on gingival microvasculature: A histological study. J Indian Soc Periodontol 15: 344-348.
31. Wyganowska-Swiatkowska M, Nohawica MM (2015) Effect of tobacco smoking on human gingival and periodontal fibroblasts-A systemic review of literature. Przegl Lek 72: 158-160.

32. Tipton DA, Dabbous MK (1995) Effects of nicotine on proliferation and extracelluar matrix production of human gingival fibroblasts in vitro. J Periodontol 66: 1056-1064.

33. Seyedmajidi M, Keshavarzi P, Bijani A, Faraji R, Babaee N (2013) A histopathological study of smoking on free gingival in patients with moderate to severe periodontitis. Caspian J Dent Res 2: 39-45.

34. Peto J (2012) The effects of smoking should be measured in pack-years: misconceptions 4. Br J Cancer 107: 406-407. 